\title{
The effect of $n$-butanol extraction of Potentilla anserina $L$. on hypoxia-induced inflammatory factors expression of wistar rat cortical neuron
}

\begin{abstract}
The study aims to investigate the effects of n-butanol extraction of Potentilla anserina L. (NP) on cortical neuronal cultures and subject to $2 \mathrm{~h}$ of oxygen deprivation hypoxialike injury. The investigation demonstrated that NP at 2,1 and $0.5 \mathrm{mg} / \mathrm{ml}$ effectively prevented the decrease of neuron survival rates and the increase of LDH release after exposure of cortical cultures to $2 \mathrm{~h}$-oxygen deprivation. These results indicated that NP has neuroprotective effect against neuronal cell death, induced by oxygen deprivation. High-throughout suspension array technology for simultaneous detection of multiple inflammatory factors expression showed that IL- $1 \beta$, IL- 6 and TNF- $\alpha$ participated in the neuroprotective effect against oxygen deprivation-induced neuronal death.
\end{abstract}

Keywords: Potentilla anserine $l$, neuron, hypoxia, inflammatory factors
Volume 3 Issue 4 - 2017

\author{
Jing Sun,' Hong Sheng Gao, 2,3 Guangming \\ Song,' Zhen Yang, ${ }^{3}$ Bin Meng,, Wen Li Xie,' \\ Ling Wang, ${ }^{4}$ Ling Zhi Li, ${ }^{3}$ Yong Liang Zhang ${ }^{3}$ \\ 'Department of Pharmacology, Logistic University of Chinese \\ People's Armed Police Forces, China \\ 2Department of Military Hygiene, Logistic University of Chinese \\ People's Armed Police Forces, China \\ ${ }^{3}$ Tianjin Key Laboratory of Biomarker for Occupational \& \\ Environmental Hazard, China \\ ${ }^{4}$ Department of Foreign Language, Logistic University of \\ Chinese People's Armed Police Forces, China
}

Correspondence: Hong Sheng Gao, Department of Military Hygiene, Logistic University of Chinese People's Armed Police Forces, Tianjin Key Laboratory of Biomarker for Occupational \& Environmental Hazard, China, Email ghstj@aliyun.com Yong Liang Zhang, Tianjin Key Laboratory of Biomarker for Occupational \& Environmental Hazard, China, Email 15332127078@qq.com

\section{Introduction}

Nowadays, Chinese traditional medicines are being increasingly concerned in the treatment of ischemic brain vascular diseases. The Tibetan herb of Potentilla anserina, belonging to the genus Potentilla, is widely distributed in the western areas of China. For thousands ofyears, this Tibetan traditional herb has been popularly used for replenishing qi and blood, strengthening the spleen and harmonizing the stomach .Previous studies (in vitro and in vivo) proved: the n-butanol extraction of Potentilla anserina L. (NP) showed a remarkably protective effect on hypoxia cardiomyocytes. Protective effects of NP against pituitrin-induced acute myocardial ischemic injury were also observed. ${ }^{1}$ In this study, we investigated the effect of NP on hypoxia-induced cortical neuron of rat. Furthermore, we attempted to investigate the influence of NP on inflammatory mediators of oxygen deprivation-injured cortical neurons.

\section{Materials and methods}

\section{Drugs and reagents}

The roots of Potentilla anserina L. were collected from Yu Shu, Qinghai Province of China. NP was extracted from Potentilla anserina L. as follows: the air-dried roots of Potentilla anserine $L$. were powdered and refluxed with $70 \%$ ethanol for $3 \mathrm{~h}$ (twice) and concentrated under reduced pressure. The crude extract was suspended in hot water $(\times 10)$ and partitioned successively with $\mathrm{H} 2 \mathrm{O}$ saturated petroleum ether $(\times 3)$, acetic ether $(\times 3)$, n-butanol $(\times 4)$ in the same volume. The n-butanol extracts were filtered and evaporated under vaccum at $45^{\circ} \mathrm{C}$ and lyophilized and then NP was achieved.

Dulbecco's modified Eagle's medium/Ham's F-12 medium (DMEM/F12) and fetal bovine serum were purchased from GIBCO. Neurobasal media, B27 supplement were obtained from Invitrogen. Trypsin and poly-L-lysine were purchased from Sigma-Aldrich. The assay kit of lactate dehydrogenase (LDH) was purchased from Nanjing Jiancheng Co. Procarta cytokine assay kit was purchased from Panomics. All of the other reagents were analytical reagents (AR).

\section{Cortical neuron cultures}

Newborn Wistar rats within $24 \mathrm{~h}$ were obtained from Vital River Laboratories. Animal experiments were carried out in accordance with institutional guidelines and ethics. According to the procedures described previously, brain cell cultures were obtained from the cerebral cortex of newborn Wistar rats within $24 \mathrm{~h},{ }^{2,3}$ newborn rats were briefly sacrificed. Cerebral hemispheres were removed from the brain, and were dissected in ice-cold D-Hank's medium, Menings and blood vessels were meticulously removed. Following a wash step with D-Hank's medium, the brains were digested with trypsin $(0.125 \%)$ for $20 \mathrm{~min}$ at $37^{\circ} \mathrm{C}$. Then cortexes were dissociated by repeated pipetting, and filtered through a nylon sieve (200 mesh, hole width 95um). Cells were plated at a density of $5^{*} 105 / \mathrm{ml}$ on L-polylysin-coated dishes, 
and maintained in Dulbecco's Modified Eagle's Medium/Ham's F-12 medium (DMEM/F12) supplemented with $10 \%$ fetal bovine serum, penicillin(100units $/ \mathrm{ml}$ ), streptomycin $(0.1 \mathrm{mg} / \mathrm{ml})$, containing $10 \mathrm{mM}$ glucose. The cultures were replenished with Neurobasal media, supplemented with 2\%B-27, 1 and 4days after plating. 8days after plating, the cultures were used for the experiments.

\section{Preparation of oxygen deprivation model}

Here we produced chemical anoxia by using sodium dithionite (Na2S2O4), which is capable of scavenging $\mathrm{O} 2$ molecules in solution and reducing the oxygen tension (PO2). This method has been used successfully in inducing anoxia in cells. ${ }^{4,5}$

Cells were randomly divided into 6 groups: control group (control); oxygen deprivation group (model), NP $(2,1,0.5 \mathrm{mg} / \mathrm{mL})$ groups. NP was given at the start of oxygen deprivation phase, acting through the processes of oxygen deprivation. After the Neurons were washed with D-Hank's medium, the culture media were replaced with serumfree DMEM/F12 medium containing different concentrations of NP. $15 \mathrm{~min}$ later a deoxygenated reagent $10 \mathrm{mmol} / \mathrm{L}$ sodium dithionite (Na2S2O4) was put in for $2 \mathrm{~h}$. Control group was only treated with serum-free DMEM/F12 medium.

\section{Assessment of neuronal viability}

Neuronal survival rate was analyzed according to the method previously reported. ${ }^{2}$ Neuron death was determined by measuring reduction of 3-(4,5-dimethylthiazol-2-yl)-2,5-diphenyltetrazolium bromide(MTT). Briefly, after oxygen deprivation, MTT was added to all those assays at a final concentration of $0.5 \mathrm{mg} / \mathrm{ml}$ for 4 hours at $37^{\circ} \mathrm{C}$. The amount of MTT formazan was dissolved by DMSO, quantified by determining calorimetrically its absorbance at $570 \mathrm{~nm}$ using a microplate reader, the percentage viability was calculated.

\section{Lactate dehydrogenase efflux assay}

The release of Lactate Dehydrogenase (LDH) was measured from culture medium using an LDH assay kit according to the reported method. ${ }^{3}$ Briefly, the culture medium and cell samples which were solubilized with $0.1 \%$ Triton X-100 were collected and incubated with a substrate solution containing nitro blue tetrazolium, diaphorase, and $\mathrm{NAD}+$ at $37^{\circ} \mathrm{C}$ for $45 \mathrm{~min}$. Then the reaction was terminated with a stop solution $(0.5 \mathrm{M} \mathrm{HCL})$. The absorbance measurements at $550 \mathrm{~nm}$ were taken as LDH release. Release LDH was calculated as the percentage of LDH in the medium vs. total LDH activity (cells plus medium).

\section{High-throughout suspension array technology for simultaneous detection of multiple inflammatory factors expression}

After exposure to oxygen deprivation, cortical neurons were collected by scrapping and low-speed centrifugation $(1,000 \mathrm{rpm}$, $10 \mathrm{~min})$. Cell pellets were lysed at $4^{\circ} \mathrm{C}$ for $30 \mathrm{~min}$ in a buffer containing as follows: Tris- $\mathrm{HCl}(20 \mathrm{mM}), 150 \mathrm{mM} \mathrm{NaCl}, 1 \mathrm{mM}$ EGTA, $1 \mathrm{mM}$ EDTA, 1mM phenylmethylsulfonyl fluoride (PMSF), 1\% NONIDET $\mathrm{P}-40,0.1 \%$ aprotinin, $0.7 \mathrm{mg} / \mathrm{ml}$ pepstatin, and $1 \mu \mathrm{g} / \mathrm{ml}$ leupeptin. Those samples were cleared by centrifugation of $12,000 \mathrm{rpm}$ at $4^{\circ} \mathrm{C}$, and the supernatants were used for high-throughout suspension array. The release of inflammatory factors was measured by using a Procarta cytokine assay kit.

\section{Data analysis and statistics}

Data were presented as means $\pm \mathrm{SD}$. Statistical analysis was performed by using SPSS 11.5 software. There is significant differences when $\mathrm{p}<0.05$.

\section{Results}

\section{Cortical neuron viability}

Cortical neurons extended multiple neurites per neuron with increasing neurite outgrowth over the first week in culture (Figure 1). Relative to normal group, neuron survival rates had significant changes in oxygen deprivation group, and decreased to $23.1 \pm 3.2 \%$ $(n=6)$ Compared with oxygen deprivation group, high, middle and low concentration of NP treatment increased cell survival rate to $61.7 \pm 6.1 \%, 55.0 \pm 5.0 \%$ and $36.7 \pm 3.7 \%(n=6$, Figure 2$)$

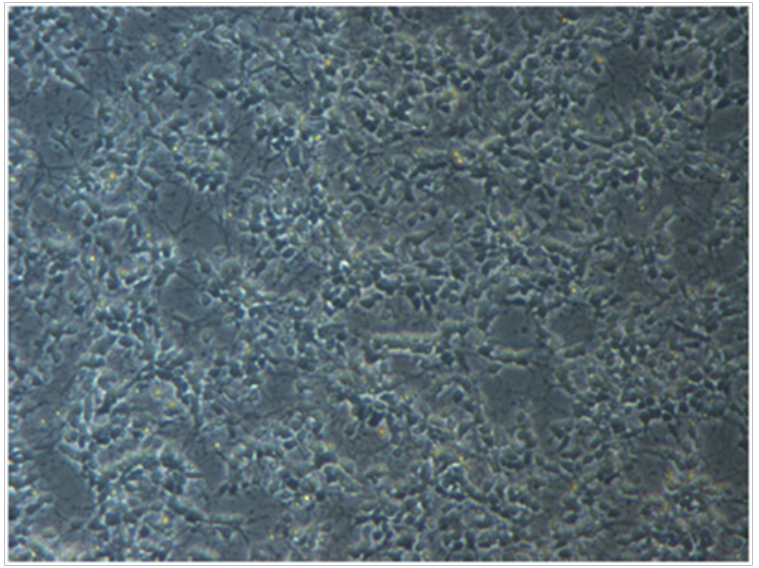

Figure I Photomicrographs of cultured cortical neurons. Cortical neurons extended multiple neurites per neuron with increasing neurite outgrowth over the first week in culture $(200 x)$.

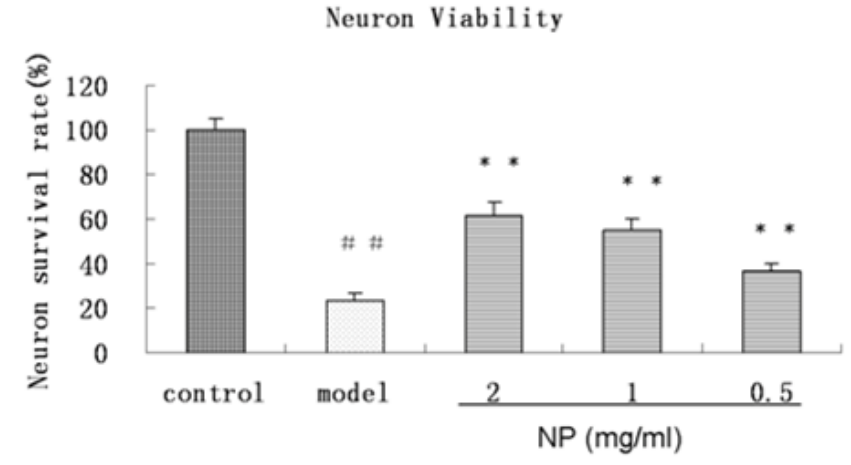

Figure 2 Effects of PN on the viability of oxygen deprivation injured cortical neuron. Viability of the neuron without oxygen deprivation injury is defined as $100 \%$, Means $\pm S D, n=6$. \# $<<0.01$ vs control, ${ }^{*} p<0.01$ vs \pm model (oxygen deprivation).

\section{Effects of PN on the release of LDH of oxygen deprivation injured cortical neurons}

As shown in Figure 3, the percentage of LDH level in the medium of control neuronal cultures was $16.4 \pm 6.6 \%$. After oxygen deprivation, the percentage of $\mathrm{LDH}$ in the medium was increased to $57.9 \pm 8.5 \%(n=6, p<0.01)$. Compared with oxygen deprivation group, the release of LDH in the medium had significant changes in PN treated groups and decreased to $36.5 \pm 4.1 \%(n=6, p<0.01)$

\section{Effects of PN on the expression of inflammatory factors of oxygen deprivation injured cortical neurons}

Our results show that the IL-6 is decreased in cortical neurons after exposure to oxygen deprivation (by $90 \%$ approximately, $\mathrm{P}<0.001$ ) it 
is not significantly prevented by NP ( $\mathrm{p}>0.05)$ (Figure 4A). TNF- $\alpha$ and IL-1 $\beta$ decrease as well after exposure to oxygen deprivation (by $73 \%$ and $70 \%$ separately). Exposure to NP partly prevents these decrease in neuronal cultures (TNF- $\alpha$ 57.1 \pm 8.8 vs $20.2 \pm 5.3$; IL- $1 \beta 29.7 \pm 4.9$ vs 17.6 \pm 4.8 ) (Figure 4B) (Figure 4C).

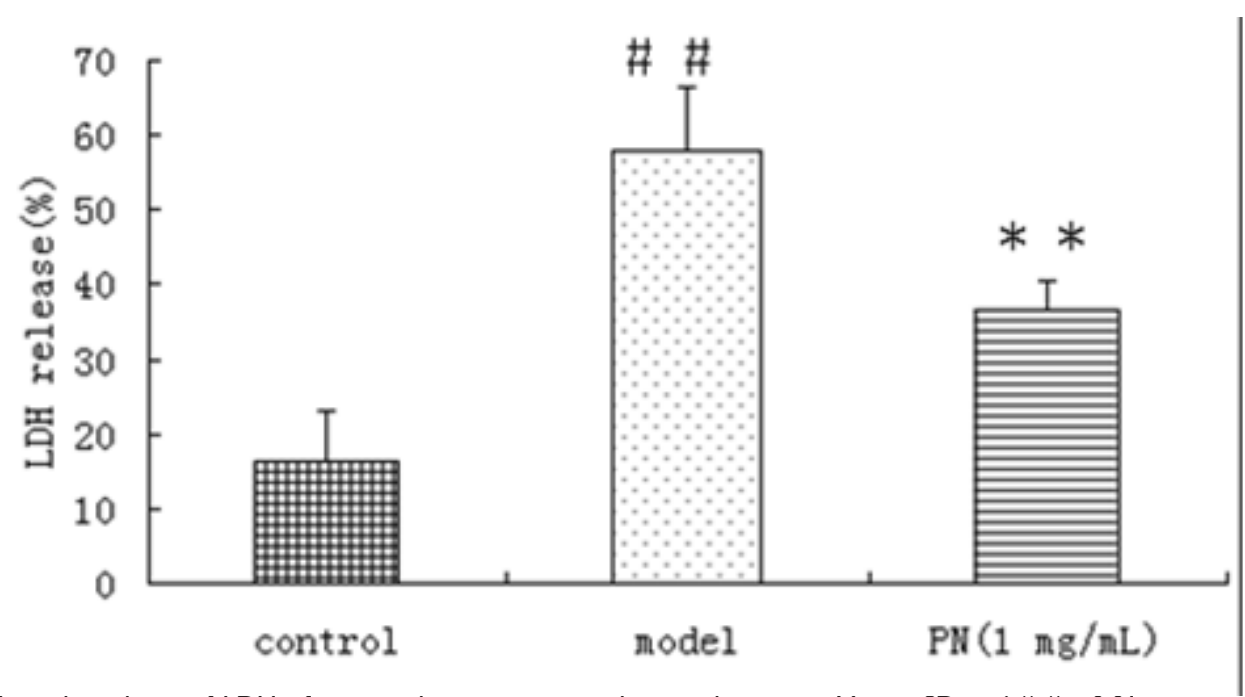

Figure 3 Effects of $\mathrm{PN}$ on the release of $\mathrm{LDH}$ of oxygen deprivation injured cortical neurons. Means $\pm \mathrm{SD}, \mathrm{n}=6 . \# \# \mathrm{p}<0.01$ vs control, **p $<0.0 \mathrm{l}$ vs \pm model (oxygen deprivation).

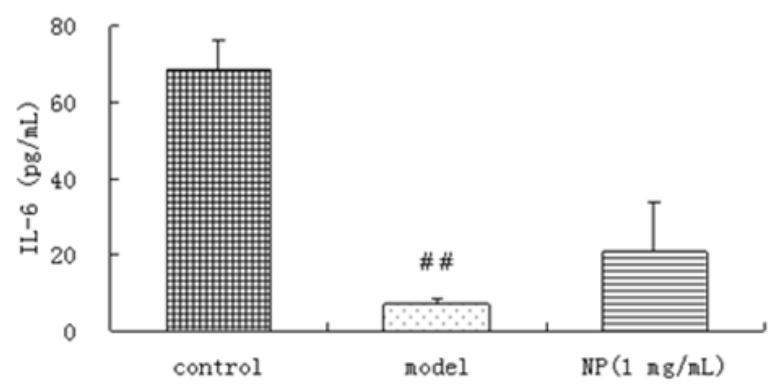

Figure 4A

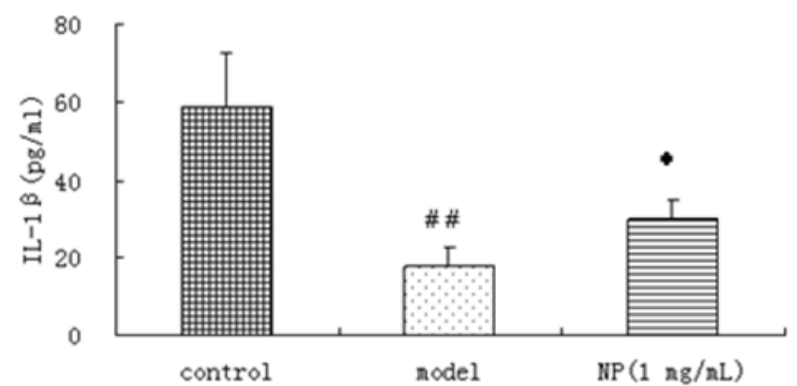

Figure 4B

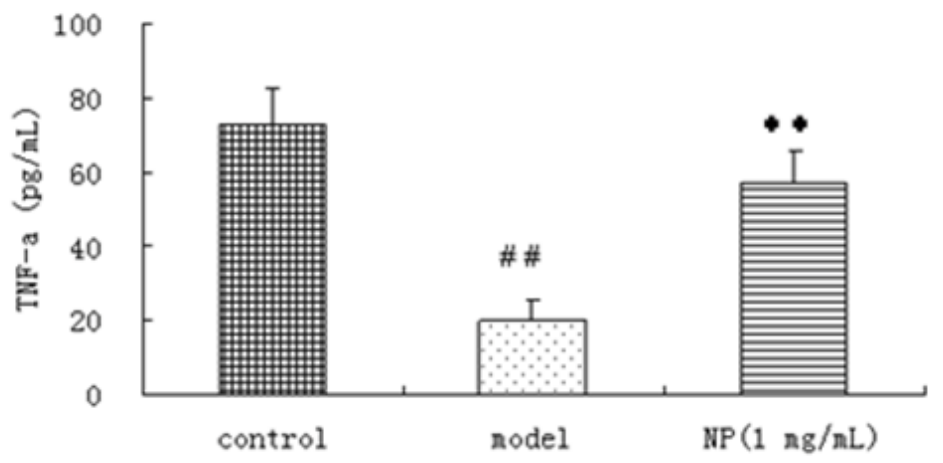

Figure 4C

Figure 4 Effects of PN on the expression of inflammatory factors IL-6、TNF- $\alpha$ and IL-I $\beta$ of oxygen deprivation injured cortical neurons. Means \pm SD, $n=6$. \# $\# p<0.01$ vs control, ${ }^{* *} p<0.01$ vs \pm model, ${ }^{*} p<0.05$ vs \pm model (oxygen deprivation).

\section{Discussion}

It has been previously reported that the n-butanol extraction of Potentilla anserina $L$. (NP) showed a remarkably protective effect on hypoxia cardiomyocytes in vitro and in vivo. ${ }^{6-8}$ The present study showed NP to be a promising neuro protection against hypoxia damage, however, its mechanism of action has not been well elucidated. Therefore, we examined the direct effects of NP on cortical neuronal cultures subjected to $2 \mathrm{~h}$ of oxygen deprivation hypoxia-like injury and aimed to investigate the potential mechanisms of this drug for its therapeutic intervention before hypoxia.

Using rat cortical neurons, our study is the first to demonstrate that NP protects neurons from oxygen deprivation mediated neurotoxicity. After exposure of cortical cultures to 2h-oxygen deprivation, the decrease of neuronal viability along with the increase of LDH release 
were detected. It is suggested that $2 \mathrm{~h}$-oxygen deprivation caused serious damage to these cultures. NP effectively prevented the decrease of neuron survival rates and the increase of LDH release. These results indicated that NP exerted some effects on the damage of $2 \mathrm{~h}$-oxygen deprivation in cortical cultures.

Hypoxia and inflammation are intertwined at the molecular, cellular, and clinical levels. ${ }^{9}$ In neurodegenerative pathologies, inflammation has always been considered detrimental. However, emerging evidence suggests that some components of the neuro inflammatory response are likely to be critical mediators of repairing. From studies in ischemic brain damage, it is clear that the same inflammatory mediators (IL-1 $\beta$, IL-6 and TNF-a) exacerbated by brain damage have also been shown to induce neuroprotection. ${ }^{10}$

IL-6 is a multifunctional cytokine with major roles in the immune, hematopoietic, and nervous systems. ${ }^{11,12}$ Both of the studies in vivo and in vitro indicate that IL-6 mediates neuroprotective activity. ${ }^{13}$ Treatment with IL-6 increases the survival of retinal ganglion cells in vitro ${ }^{14}$ and protects cerebellar granule cells ${ }^{15}$ and neuroblastoma cells in culture ${ }^{16}$ against glutamate-induced toxicity and oxidative damage. Moreover, injection of IL-6 reduces the volume of induced brain infarcts in rats and protects against N-methyl-D-aspartate-induced toxicity in cortical, striatal, and retinal neurons. ${ }^{17}$ According to our experiments, IL-6 is decreased in cortical neurons after exposure to oxygen deprivation (by $90 \%$ approximately, $\mathrm{P}<0.001$ ) it is not significant to be prevented by NP $(\mathrm{p}>0.05)$.

The experiments in which endogenous TNF- $\alpha$ bioactivity is blocked confirm that this cytokine is a critical mediator to confer neuro protection in vitro model of ischemia. Other results also report protective properties of TNF- $\alpha$ against metabolic poisoning in astrocytes, PC6 cells and neurones. ${ }^{18}$ The same laboratory has demonstrated that TNF receptor deficient mice (TNFRKO) show much greater neuronal death in response to excitotoxic or ischemic insults. ${ }^{19}$ Moreover, TNF- $\alpha$ up-regulation of the NF-kh-target genes initiate protective pathways against apoptosis in neuronal and in nonneuronal cells. ${ }^{19-21}$

Neuroprotection against insults to the CNS has been increasingly attributed to different immune mediators, with an emerging view of a pleiotropic action, from a detrimental or inflammatory effect to a protective mode of action. ${ }^{18-24}$ We have made the observations that NP is neuroprotective against neuronal cell death, induced by oxygen diprivation and it has been shown that in neurons, IL-1 $\beta$, IL-6 and TNF-a may be an important component of neuroprotection against oxygen deprivation-induced neuronal death.

\section{Acknowledgments}

This work was supported by the National Nature Science Foundation of the People's Republic of China, No. 81073152 and the National Science Foundation for Post-doctoral Scientists of China, No.20100470106.

\section{Conflict of interest}

Author declares that there is no conflict of interest.

\section{References}

1. Li JY, Li Y, Gong HY, et al. Protective effects of n-butanol extract of Potentilla anserina $L$. on acute myocardial ischemic injury in mice. Journal of Chinese Integrative Medicine. 2009;7(1):48-52.

2. Ban JY, Jeon SY, Nguyen TT, et al. Neuroprotective effect of oxyresveratrol from smilacis chinae rhizome on amyloid Beta protein (25-35)-induced neurotoxicity in cultured rat cortical neurons. Biol Pharm Bull. 2006;29(12):2419-2424.
3. Liu R, Gao M, Yang ZH, et al. Pinocembrin protects rat brain against oxidation and apoptosis induced by ischemia-reperfusion both in vivo and in vitro. Brain Res. 2008;1216:104-115.

4. Jiang RG, Eyzaguirre C. Effects of hypoxia and putative transmitters on [Ca2+]I of rat glomus cells. Brain Res. 2004;995(2):285-296.

5. Punn A, Mockridge JW, Farooqui S, et al. Sustained activation of p42/ p44 mitogen-activated protein kinase during recovery from simulated ischaemia mediates adaptive cytoprotection in cardiomyocytes. Biochem J. 2000;350(pt 3):891-899.

6. Li JY, Li LZ, Zhang YL, et al. Protective effect of Poteutilla anserina L. on hypoxia injury in neonatal rats cardiomyocytes. Chin New Drug J. 2007;16(12):944-946.

7. Ye L, Chen Y, Li LZ. Protective effect of alcohol extract of Poteutilla anserina $L$. on acute myocardial ischemin/reperfusion injury in mice. Chin Tradit Herbal Drugs. 2009;40:774-777.

8. Lv Q, Qin XJ, Zhang XN, et al. Effects of Poteutilla anserina L. on acute ischemic myocardiac muscle and the differentially expressed proteins in serum in rats. Acta Academiae Medicinae CPAF. 2011;20:429-422.

9. Eltzschig HK, Carmeliet P. Hypoxia and inflammation. $N$ Engl J Med. 2011;364(7):656-665.

10. Barone FC, Feuerstein GZ. Inflammatory mediators and stroke: new opportunities for novel therapeutics. J Cereb Blood Flow Metab. 1999;19(8):819-834.

11. Kamimura D, Ishihara K, Hirano T. IL-6 signal transduction and it physiological roles: the signal orchestration model. Rev Physiol Biochem Pharmacol. 2003;149:1-38.

12. Van Wagoner NJ, Benveniste EN. Interleukin-6 expression and regulation in astrocytes. J Neuroimmunol. 1999;100(1-2):124-139.

13. Gadient RA, Otten UH. Interleukin-6 (IL-6)-a molecule with both beneficial and destructive potentials. Prog Neurobiol. 1997;52(5):379-390.

14. Mendonca Torres PM, de Araujo EG. Interleukin-6 increases the survival of retinal ganglion cells in vitro. J Neuroimmunol. 2001;117(1-2):43-50.

15. Peng YP, Qiu YH, Lu JH, et al. Interleukin-6 protects cultured cerebellar granule neurons against glutamate-induced neurotoxicity. Neurosci Lett. 2005;374(3):192-196.

16. Bissonnette CJ, Klegeris A, McGeer PL, et al. Interleukin 1alpha and interleukin 6 protect human neuronal SH-SY5Y cells from oxidative damage. Neurosci Lett. 2004;361(1-3):40-43.

17. Ali $\mathrm{C}$, Nicole $\mathrm{O}$, Docagne $\mathrm{F}$, et al. Ischemia-induced interleukin-6 as a potential endogenous neuroprotective cytokine against NMDA receptor-mediated excitotoxicity in the brain. $J$ Cereb Blood Flow Metab. 2000;20(6):956-966.

18. Bruce Keller AJ, Geddes JW, Knapp PE, et al. Anti-death properties of TNF against metabolic poisoning: mitochondrial stabilization by MnSOD. J Neuroimmunol. 1999;93(1-2):53-71.

19. Bruce AJ, Boling W, Kindy MS, et al. Altered neuronal and microglial responses to excitotoxic and ischemic brain injury in mice lacking TNF receptors. Nat Med. 1996;2(7):788-794.

20. Marchetti L, Klein M, Schlett K, et al. Tumor necrosis factor (TNF)mediated neuroprotection against glutamate- induced excitotoxicity is enhanced by N-methyl-d-aspartate receptor activation. Essential role of a TNF receptor 2-mediated phosphatidylinositol 3-kinase-dependent NF-kappa B pathway. J Biol Chem. 2004;279(31):32869-32881.

21. Barger SW, Horster D, Furukawa K, et al. Tumor necrosis factors alpha and beta protect neurons against amyloid beta-peptide toxicity: evidence for involvement of a kappa B-binding factor and attenuation of peroxide and $\mathrm{Ca} 2+$ accumulation. Proc Natl Acad Sci USA. 1995;92(20):9328-9332. 
22. Mattson MP, Goodman Y, Luo H, et al. Activation of NF-kappa $\mathrm{B}$ protects hippocampal neurons against oxidative stress-induced apoptosis: evidence for induction of manganese superoxide dismutase and suppression of peroxynitrite production and protein tyrosine nitration. J Neurosci Res. 1997;49(6):681-697.

23. Lastres Becker I, Cartmell T, Molina Holgado F. Endotoxin preconditioning protects neurones from in vitro ischemia: Role of endogenous IL-1 $\beta$ and TNF- $\alpha . J$ Neuroimmunol. 2006;173(1-2):108-116
24. Westberg JA, Serlachius M, Lankila P, et al. Hypoxic preconditioning induces neuroprotective stanniocalcin-1 in brain via IL-6 signaling. Stroke. 2007;38(3):1025-1030. 